\title{
Integrity Monitoring Using ARAIM for Bridging DFMC SBAS Outages in Road Transport
}

\author{
Kan Wang ${ }^{1}$, Ahmed El-Mowafy ${ }^{1}$, Jizhong $\mathrm{Wu}^{2}$ \\ ${ }^{1}$ School of Earth and Planetary Sciences, Curtin University, Australia \\ ${ }^{2}$ School of Geomatics Science and Technology, Nanjing Tech University, China
}

\section{BIOGRAPHIES}

Kan Wang is a research fellow in the School of Earth and Planetary Sciences, Curtin University. She obtained her doctoral degree in GNSS advanced modelling from ETH Zurich in 2016. Her research interest includes high-accuracy GNSS positioning, integrity monitoring, SBAS, RTK and PPP-RTK processing.

Ahmed El-Mowafy is Assoc. Professor of Positioning and Navigation and Director of Graduate Research, School of Earth and Planetary Sciences, Curtin University, Australia. He obtained his Ph.D. from the University of Calgary, Canada. He has extensive publications in precise positioning and navigation using GNSS, quality control, integrity monitoring and estimation theory.

Jizhong Wu is an Associate Professor at Nanjing Tech University, China. He received his Ph.D. in geodesy and surveying engineering in 2012. His main research interests include precise GNSS positioning and the application of GNSS to surveying engineering.

\begin{abstract}
The new generation of the satellite-based augmentation system (SBAS) has been initiated in Australia and New Zealand since 2017 and is anticipated to be a fully operational system by 2023. In addition to the traditional L1 service, the new SBAS also supports the dual-frequency multi-constellation (DFMC) service and the precise point positioning (PPP) service for GPS and Galileo dualfrequency users. Making use of the DFMC SBAS service, horizontal positioning accuracy at sub-meter to meter level can be realized in real-time depending on the measurement environment, which should benefit users of the intelligent transport system (ITS) in road transport. The integrity monitoring is essential for the ITS users to guarantee the reliability of the positioning service. During possible outage of the SBAS messages, the integrity monitoring of the DFMC SBAS positioning becomes difficult due to the missing corrections integrity information that should be updated regularly. Even when relieving these strict time-out windows for land-based applications, the increase of the horizontal protection levels (HPLs) with time is shown to be dramatic. In this contribution, a modified version of the advanced receiver autonomous integrity monitoring (ARAIM) algorithm is used during such SBAS outages. The HPLs are computed based on the ARAIM algorithm with different integrity parameters assumed to investigate their impact on the HPL. Under the expectation that higher precision of the satellite clocks and orbit corrections can be achieved for the new generations of the GPS and Galileo satellites in the future, the HPLs computed with the proposed modified ARAIM algorithm are shown to be close to the level of those based on the DFMC SBAS. In such a case, the ARAIM is expected to be a useful alternative approach for bridging the integrity monitoring of the non-safety-of-life ITS applications during the SBAS outages.
\end{abstract}

\section{INTRODUCTION}

In road transport applications, such as Intelligent Transport Systems (ITS), integrity monitoring (IM) is an essential task to guarantee the reliability of the vehicle positioning results. Under a pre-defined probability of hazardous misleading information (PHMI), set according to the application, a protection level is computed at each time epoch to bound the actual positioning errors. In the case that the required alert limit is exceeded by the protection level, a warning message is sent to the user within a pre-defined time-to-alert. 
Nowadays, high-accuracy positioning results can be obtained with different advanced positioning technics. Compared to the realtime kinematic (RTK) positioning method, which requires a reference station typically within $10 \mathrm{~km}$ from the user, standalone techniques like precise point positioning (PPP) can also provide users with the required high positioning accuracy. However, despite the long convergence time of tens of minutes that is needed for the PPP filter [1-3], the precise satellite clocks and orbits need to be obtained in real-time via, e.g., an internet link. On the other hand, satellite-based augmentation systems (SBAS) can provide users with free-of-charge precise satellite-related products. The satellite-related corrections, integrity, and range information are directly broadcast from the geostationary (GEO) satellites to the users in real-time, which enables stand-alone real-time positioning.

The traditional SBAS service augments only the L1 observations. SBAS is moving towards a newer generation, providing to dual-frequency multi-constellation (DFMC) users observation corrections and integrity information. A test-bed of the new generation of SBAS was initiated in Australia and New Zealand in 2017 in preparation for building an operational system [4]. The users eliminated the first-order ionospheric delays by forming the ionosphere-free (IF) linear combination and an improved measurement geometry are also available through the use of both GPS and Galileo signals. In a previous study [5], test results showed that using DFMC SBAS service, the horizontal positioning accuracy for road transport users can reach sub-meter to meter-level depending on the measurement environment, which realizes standalone high-accuracy positioning in real-time without any internet link. For applications with higher accuracy requirements, the new SBAS also supports the PPP service with the precise orbit and clock corrections and integrity information sent via L1 for GPS satellites and via L5 for GPS/Galileo satellites [6]. A sub-dm to dm horizontal positioning accuracy can be achieved after a certain convergence time.

Due to possible service failures or signal blockage in complicated measurement environments such as urban areas, the SBAS users may experience an outage of the precise corrections and integrity information. The satellite orbits and clocks can be predicted using different mathematical models for short time periods, e.g. less than $15 \mathrm{~min}$, with acceptable errors [5]. However, the protection levels, computed based on the ED-259 standards [7], would degrade significantly with time due to the lack of updated integrity information [5].

In this contribution, the authors exploit and assess the possibility of using the advanced receiver autonomous integrity monitoring (ARAIM) algorithm $[8,9]$ for bridging the ITS integrity monitoring during the SBAS outages. We restrict focus on the DFMC SBAS utilizing the carrier-smoothed code observations. The fault detection and exclusion (FDE) procedure and the approach to compute the HPL are explained. To assess the proposed ARAIM bridging approach, a station in Perth, Australia is used as an example. Compatible assumptions with SBAS on the integrity risk and methodology were implemented. The HPLs are computed based on different assumptions of the integrity parameters like the $\sigma_{U R A, i}$ and $\sigma_{U R E, i}$, which denote the standard deviations of the orbit and clock errors of satellite $i$ for integrity purpose and for accuracy and continuity purpose, respectively. In addition, the maximum nominal bias for integrity purpose, denoted by $b_{n o m, i}$, which plays an important role in the resulting HPLs, is tested for different possible values. During assumed breaks in SBAS, the computed HPLs based on the ARAIM algorithm are compared with those based on the actual DFMC SBAS without breaks. As the Signal-in-Space Range Error (SISRE) reduces in the new generations of GPS satellites due to the continuous improvement of the on-board satellite clock performance [10], it is expected that the $\sigma_{U R A, i}$ can be further reduced in the future. As such, the ARAIM-based HPLs assuming small values for $\sigma_{U R A, i}$ are also compared with those based on the SBAS DFMC.

\section{DEGRADATION IN THE HPL DURING SBAS OUTAGES}

FOR DFMC SBAS, after forming the IF linear combination of the carrier-smoothed code observations for both the GPS and Galileo satellite observations, the estimated unknown vector includes the 3-dimensional positional increments to the approximate position and the GPS and Galileo receiver clock errors. Assuming that the total number of the processed GPS and Galileo satellites amounts to $n$ at a single epoch, where each satellite gives one IF combination of carrier-smoothed GPS or Galileo observations, the linearized observation model can be expressed as

$$
\mathrm{E}(y)=G x
$$

where $y$ denotes the observed-minus-computed (O-C) measurement vector, $x$ is a $5 \times 1$ vector comprising the receiver position increments and the receiver clock errors for GPS and Galileo. $\mathrm{E}(\cdot)$ is the expectation operator. The design matrix $G$ is a $n \times 5$ matrix, where the $i$-th row of the matrix $G$ can be expressed as 


$$
G_{i}=\left[\mu_{i, E}, \quad \mu_{i, N}, \quad \mu_{i, U}, \quad e_{i, G}, \quad e_{i, E}\right]
$$

where $\mu_{i, E}, \mu_{i, N}$ and $\mu_{i, U}$ refer to the east, north and up components of the unit vector from the $i$-th satellite to the receiver, respectively. The element $e_{i, G}$ is set to one for the receiver clock offset of GPS measurements, and zero for Galileo observations. Similarly, $e_{i, E}$, is set to one for the corresponding Galileo receiver clock offset for Galileo observations and a zero for GPS observations.

The tested DFMC SBAS service supports users receiving GPS L1/L5 and Galileo E1/E5a signals. Due to the limited number of the GPS satellites transmitting the L5 signals, the SBAS test-bed utilized GPS L2 signal instead of those on L5 before 2019 to guarantee a good measurement geometry. The same signal constellation is also used in this study. In the future with more GPS satellites sending signals on L5, and with more Galileo satellites launched as planned, a better measurement geometry is expected.

In road transport, the horizontal positioning service is mostly of higher interest for the ITS users. Under a pre-defined PHMI for the horizontal positioning service, the HPLs can be computed for DFMC SBAS based on [7]. As defined in the ED-259 standards for the DFMC SBAS service [7], the HPL can be computed as follows

$$
H P L=K_{H} \cdot \sigma_{H}
$$

where $\sigma_{H}$ is the standard deviation (std) of the horizontal positioning error along the semi-major axis of the horizontal error ellipse, and $K_{H}$ is a scale factor computed based on the horizontal PHMI, denoted as $\mathrm{PHMI}_{\mathrm{H}}$, with

$$
K_{H}=\psi^{-1}\left(1-\frac{\mathrm{PHMI}_{\mathrm{H}}}{2}\right)
$$

where $\psi^{-1}$ is the quantile function (inverse cumulative distribution function - CDF) of a standard normal distribution.

The standard deviation of the horizontal positioning error $\sigma_{H}$ is mapped from that of the east $\left(\sigma_{E}\right)$ and north $\left(\sigma_{N}\right)$ std positioning errors, and the $\sigma_{E}$ and $\sigma_{N}$ are the square roots of the first two elements of the $Q_{x}$, the variance-covariance matrix of the estimated parameter vector. The matrix $Q_{x}$ can be computed based on the least-squares adjustment in the form

$$
Q_{x}=\left(G^{T} Q_{y}^{-1} G\right)^{-1}
$$

The variance-covariance matrix $Q_{y}$ in Eq. (5) is assumed a diagonal matrix. Its diagonal elements, for each satellite, are determined by applying the variance rule, i.e. the sum of the squared standard deviation of the residual errors for the satellite clock and orbit corrections $\left(\sigma_{D F C}\right)$, the standard deviation of the ionosphere delay residuals after forming the IF combination $\left(\sigma_{I}\right)$, which typically amounts to high-order ionosphere delay part, the standard deviation of the troposphere residuals after applying the empirical model $\left(\sigma_{T}\right)$, the standard deviation for the noise $\left(\sigma_{N}\right)$, and standard deviation for the combined multipath and antenna group delay errors $\left(\sigma_{M}\right)$. The detailed procedure for the FDE process and for computing the HPLs are described in [5, 7].

While the other standard deviations mentioned above are elevation-dependent functions, the $\sigma_{D F C}$, which plays an important role in computing the final HPLs [5], is closely related to the regularly updated integrity parameters broadcast by the SBAS-GEO satellite. During an outage of the SBAS messages of, e.g., $15 \mathrm{~min}$, when relying on the aviation standards as defined in [7], the corrections and integrity parameters would not be usable anymore after the corresponding time-out windows. As an example, the dual-frequency range error indicator (DFREI), an important integrity parameter to compute the $\sigma_{D F C}$, has a time-out window of less than $20 \mathrm{~s}$ for different approaches in aviation.

In road transport, even when relieving the strict time-out windows defined for aeronautical applications, the degradation parameters given in the Message Type 37 of the DFMC SBAS could also lead to a quick increase in $\sigma_{D F C}$ and the resulting HPLs. As shown in our previous study [5], during an outage of the SBAS messages of $15 \mathrm{~min}$, the degradation of the HPLs could be significant even under a good measurement geometry. As an example using the station CUT0 in Perth, Australia, as shown in Figure 1 , with the time-out windows of diverse parameters ignored and assuming a horizontal PHMI of $10^{-5}$, the HPLs that are considered for road transport applications could increase to over $15 \mathrm{~m}$ after a service outage of $15 \mathrm{~min}$ applying the last available degradation parameters. If the time-out windows of diverse parameters are correctly considered, the HPLs can theoretically not be produced after these time-out windows. 


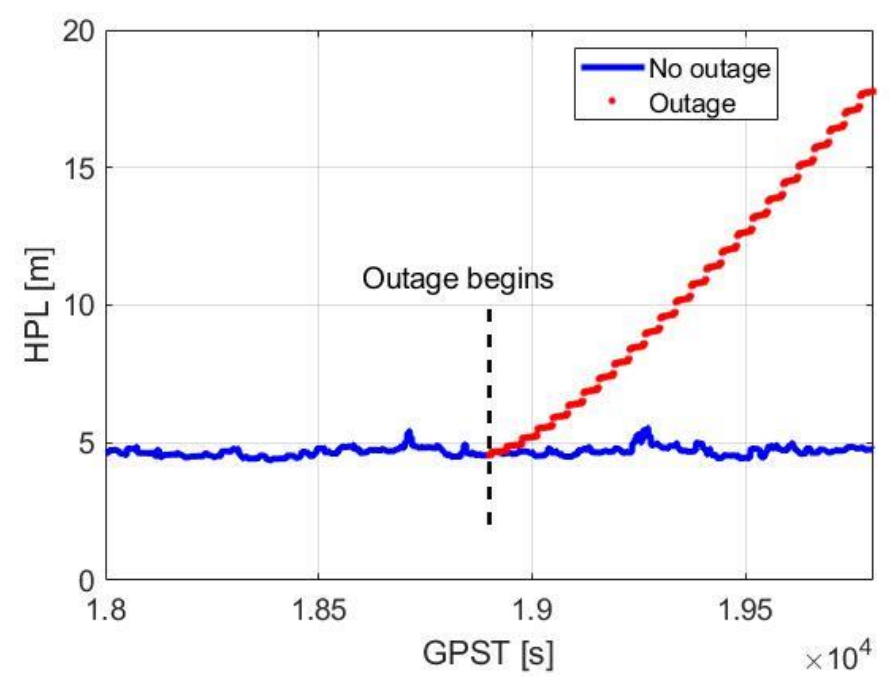

Fig 1. HPLs of the station CUT0 with DFMC SBAS messages (blue) and during the assumed SBAS outage (red). The data between 5:00 and 5:30 in GPST, 14/09/2018 was used for the plot.

\section{ARAIM ALGORITHM DURING DFMC SBAS OUTAGE}

Instead of using the last-updated integrity parameters during an SBAS outage, the ARAIM algorithm can be used instead. With the same observation equation given in Eq. (1), a weighted least-squares adjustment can be performed with all satellites in view as:

$$
\hat{x}_{0}=S_{0} y
$$

where $S_{0}=\left(G^{T} W G\right)^{-1} G^{T} W . W$ is a diagonal weighting matrix, and the $i$-th diagonal element of $W$ reads

$$
W_{i}=\frac{1}{\sigma_{U R A, i}^{2}+\sigma_{i, u s e r}^{2}+\sigma_{i, t r o p o}^{2}}
$$

for which the term $\sigma_{U R A, i}$ denotes the standard deviation of the clock and ephemeris error used for integrity, $\sigma_{i, u s e r}$ is the elevationdependent standard deviation of the user noise and multipath, and $\sigma_{i, t r o p o}$ stands for the standard deviation of the residual tropospheric delay. Detailed models for these terms can be found in [8], and the multipliers for the IF combination are considered in $\sigma_{i, u s e r}^{2}$. It should be noticed that for users in the road transport may experience more complicated multipath environments. The ground-based models for the user noise and multipath will be studied in a future work, but are not further attempted in this contribution.

During integrity monitoring, the ARAIM baseline algorithm performs the FDE procedure with the solution separation test (SST) before computing the protection levels. It compares the positional differences between the all-in-view (Eq. 6) and the subset solutions of fault mode $k$ with a test threshold. In this contribution, the $\mathrm{PHMI}_{\mathrm{H}}$ for road transport is assumed to be $10^{-5}$, and we consider only the single-satellite fault with the prior satellite fault probability $P_{\text {sat }}$ set to $10^{-5}$ per approach based on [11] and defined in the simulation settings in [9]. The probability of two or more faulty satellites amounts to about $10^{-8}$ for 15 satellites [8], and thus it is far below the assumed $\mathrm{PHMI}_{\mathrm{H}}$. As such, it is ignored in computing the HPLs. Note that a constellation-wide fault is not protected in this study. In Table 1, the parameters used for the ARAIM algorithm are given for demonstration purpose. Some of them will be explained later in this section. In road transport applications, multipath and poor satellite geometry may lead to a reduced continuity compared to aviation. The continuity requirement is dependent on the application at hand, and since this has not been defined yet for road transport, a higher continuity budget (than in aviation) of $4.2 \times 10^{-6}$ is assumed for the demonstration purpose in this article. The allocation to disruptions due to false alert is assumed to be $2 \times 10^{-6}$ in both the horizontal and vertical directions. Its allocation to the $\chi^{2}$-test, applied in the observation domain for detection of observations outliers, is assumed to be $2 \times 10^{-7}$. 
Table 1. The parameters assumed for the ARAIM algorithm. Note that $P_{S a t}, \sigma_{U R A, i}, \sigma_{U R E, i}$ and $b_{n o m, i}$ should be taken from the Integrity Support Message (ISM) when available. The values given in this table are used for demonstration purpose.

\begin{tabular}{lll}
\hline Name & Description & Tested Values \\
\hline PHMI $_{\mathrm{H}}$ & Horizontal hazardous misleading information & $10^{-5}$ \\
$P_{s a t}$ & Prior satellite fault probability & $10^{-5}$ \\
$P F A_{H}$ & Probability of false alert allocated in the horizontal direction & $2 \times 10^{-6}$ \\
$P F A_{V}$ & Probability of false alert allocated in the vertical direction & $2 \times 10^{-6}$ \\
$P F A_{\chi}{ }^{2}$ & Probability of false alert allocated to the $\chi^{2}$-test & $2 \times 10^{-7}$ \\
$\sigma_{U R A, i}$ & Standard deviation of the orbit and clock errors of satellite $i$ for integrity & $1 \mathrm{~m}, 1.5 \mathrm{~m}, 2 \mathrm{~m}, 2.5 \mathrm{~m}, 3 \mathrm{~m}$ \\
$\sigma_{U R E, i}$ & Standard deviation of the orbit and clock errors of satellite $i$ for accuracy & $2 / 3 \times \sigma_{U R A, i}$ \\
& and continuity & $0.5 \mathrm{~m}, 0.75 \mathrm{~m}, 1 \mathrm{~m}, 1.5 \mathrm{~m}$ \\
\hline
\end{tabular}

Assuming that the total number of fault modes is $N$, for the $k$-th fault mode, the corresponding $S_{k}$ is computed as

$$
S_{k}=\left(\left(B_{k} G\right)^{T} W\left(B_{k} G\right)\right)^{-1}\left(B_{k} G\right)^{T} W
$$

where $B_{k}$ is an identity matrix with the diagonal element corresponding to the suspected faulty satellite replaced by zero, i.e. excluding it from processing. The $q$-th element $(q=1,2,3)$ of the positioning solution for the $k$-th fault mode is

$$
\hat{x}_{k, q}=S_{k, q} y
$$

where $S_{k, q}$ denotes the $q$-th row of $S_{k}$. For consistency of ARAIM with DFMC SBAS procedure, where testing is performed along the semi-major axis of the horizontal error ellipsoid as the maximum error, we modified the ARAIM baseline algorithm such that the threshold for the positioning difference between the all-in-view and the subset solution $(k)$ is expressed as [7, 12]:

$$
\begin{gathered}
T_{k, H}=K_{f a, H} \times \sqrt{E_{k}^{T}\left(S_{k,(1,2)}-S_{0,(1,2)}\right) W_{U R E}^{-1}\left(S_{k,(1,2)}-S_{0,(1,2)}\right)^{T} E_{k}} \\
T_{k, V}=K_{f a, V} \times \sqrt{\left(S_{k, 3}-S_{0,3}\right) W_{U R E}^{-1}\left(S_{k, 3}-S_{0,3}\right)^{T}}
\end{gathered}
$$

where $W_{U R E}$ is a diagonal matrix derived from Eq. (7) by replacing $\sigma_{U R A, i}$ with $\sigma_{U R E, i}$, the standard deviation of the clock and ephemeris error for accuracy and continuity. $T_{k, H}$ denotes the threshold for the horizontal component along the semi-major axis for the horizontal error ellipse of $Q_{k}=S_{k,(1,2)} W^{-1} S_{k,(1,2)}^{T} . T_{k, V}$ is the threshold for the vertical component. $E_{k}$ stands for the first eigenvector of $Q_{k}$, projecting the positioning errors from the east and north directions to the direction of the semi-major axis of the error ellipse for $Q_{k}$. The sub-index $(1,2)$ denotes the first two rows in the corresponding matrix. Note that the first eigenvector for the variance-covariance matrix of the horizontal positioning differences is different from that for the horizontal positioning estimates, i.e., the $E_{k}$ defined above. The $E_{k}$ is used here to unify the direction of $T_{k, H}$ with the one that will be used for computation of the HPL. $K_{f a, H}$ and $K_{f a, V}$ are computed with the probability of false alert allocated in the horizontal $\left(P F A_{H}\right)$ and the vertical directions $\left(P F A_{V}\right)$, respectively:

$$
\begin{aligned}
& K_{f a, H}=\psi^{-1}\left(1-\frac{P F A_{H}}{2 N}\right) \\
& K_{f a, V}=\psi^{-1}\left(1-\frac{P F A_{V}}{2 N}\right)
\end{aligned}
$$

For each fault mode, the following SST is performed with the passing criterion: 


$$
\begin{gathered}
\left|\hat{x}_{k, H}-\hat{x}_{0, H}\right| \leq T_{k, H} \\
\left|\hat{x}_{k, 3}-\hat{x}_{0,3}\right| \leq T_{k, V}
\end{gathered}
$$

where $\hat{x}_{k, H}$ (including $k=0$ for the case of all-in-view satellites, corresponding to the no-fault mode, or the null hypothesis) is calculated with $E_{k}^{T} \hat{x}_{k,(1,2)}$. If the test fails for any satellite observations, those faulty satellites are excluded, and the SST is re-applied until it passes for all remaining satellites.

After passing the SST, which is performed in the position-parameter domain, a $\chi^{2}$-test is performed in the observation domain. The two tests are performed in orthogonal directions, and therefore, there is a very small chance that some tests detected by one test is missed by the other. For more details see [13]. In the $\chi^{2}$-test, the statistic $T_{\chi^{2}}$ is compared with the threshold $Q_{\chi^{2}, f}^{-1}\left(1-P F A_{\chi^{2}}\right)$, which denotes the inverse CDF of the $\chi^{2}$-distribution with $(f)$ degrees of freedom. The test statistic is

$$
T_{\chi^{2}}=y^{T}\left(W_{U R E}-W_{U R E} G\left(G^{T} W_{U R E} G\right)^{-1} G^{T} W_{U R E}\right) y
$$

The test passing criterion is $T_{\chi^{2}}<Q_{\chi^{2}, f}^{-1}\left(1-P F A_{\chi^{2}}\right)$. The observations in the accepted HPL, computed later, should pass both the SST and the $\chi^{2}$ test.

The HPL for the fault mode $k$, which is aligned in the direction of the semi-major axis of the horizontal error ellipse of $Q_{k}$, can be expressed as

$$
H P L_{k}=T_{k, H}+K_{k} \times \sigma_{k}+\left|E_{k}^{T} \cdot S_{k,(1,2)}\right| \times b_{n o m}
$$

and the HPL for all satellites in view is computed with:

$$
H P L_{0}=K_{0} \times \sigma_{0}+\left|E_{0}^{T} \cdot S_{0,(1,2)}\right| \times b_{n o m}
$$

where $\sigma_{k}=\sqrt{E_{k}^{T} Q_{k} E_{k}}$ (including $k=0$ ). Recall that the sub-index $(1,2)$ refers to the first two rows of the corresponding matrix. $b_{\text {nom }}$ is the maximum nominal bias vector for the integrity purpose, containing the maximum nominal biases of satellite $i\left(b_{\text {nom, } i}\right)$ as elements. It is here projected into the direction along the semi-major axis of the horizontal error ellipse. $K_{k}$ and $K_{0}$ are scalar factors expressed as:

$$
\begin{gathered}
K_{k}=\psi^{-1}\left(1-\frac{\text { PHMI }_{\mathrm{H}}}{(N+1) P_{\text {sat }}}\right) \\
K_{0}=\psi^{-1}\left(1-\frac{\mathrm{PHMI}_{\mathrm{H}}}{2(N+1)}\right)
\end{gathered}
$$

Herein, it is assumed that $\mathrm{PHMI}_{\mathrm{H}}$ is equally allocated for all modes ( $N$ fault modes + the all-in-view mode), and the probability of the not monitored faults is ignored as it is far below the $\mathrm{PHMI}_{\mathrm{H}}$ assumed in this study. An upper bound of the HPL can then be computed as the maximum of the corresponding HPLs for all the subset modes $\left(H P L_{k}\right)$ and the all-in-view mode $\left(H P L_{0}\right)[14,14]$. 


\section{TEST RESULTS}

In this section, the continuous operating reference station (CORS) CUT0 located in the Curtin University, Perth, Australia was used for demonstration of the changes in HPLs when SBAS is used and when the modified ARAIM is utilized to bridge possible breaks in SBAS. Two different time periods on September 14, 2018, were used for the processing, i.e., from 1:00 to 1:30 and from 5:00 to 5:30 in GPST. In this study, the HPLs is computed with differently assumed integrity parameters, including also those expected in the future, to study their impact on future HPLs. The HPLs are only computed using the measurement geometry without using real observations.

In the first test scenario, the SBAS messages are assumed to be available during the entire test periods, and the corresponding HPLs are illustrated with blue dots in Figures 2 and 3. In the second test scenario, the SBAS messages are assumed to experience outages from the middle of the two test periods, i.e., from 1:15 to 1:30 and from 5:15 to 5:30 in GPST. The HPLs based on the proposed ARAIM algorithm are illustrated with the red, green, magenta and cyan lines in the Figures 2 and 3, depending on the values of $\sigma_{U R A, i}, \sigma_{U R E, i}$ and $b_{n o m, i}$ used for the processing. As stated in [9], the parameters $\sigma_{U R A, i}, \sigma_{U R E, i}$ and $b_{n o m, i}$ can be derived, for instance, from the integrity support message (ISM). In studies over the past decade, different values were used for these parameters for simulation and demonstration purposes. Based on $[9,14,16,17]$, here we test a range of different values for $\sigma_{U R A, i}$ and $b_{n o m, i}$ as given in Table 1. The parameter $\sigma_{U R E, i}$ is assumed to be $2 / 3$ of the $\sigma_{U R A, i}$ as given in the simulation settings in [9]. To show the variation of the HPLs with the $\sigma_{U R A, i}$ and $b_{n o m, i}$, the test details of Figures 2 and 3 are given in Table 2.

Table 2. Test values for $\sigma_{U R A, i}$ and $b_{n o m, i}$ used in the Figures 2, 3 and 4.

\begin{tabular}{ccccc}
\hline Figure No. & GPST (DFMC SBAS) & GPST (ARAIM) & $\sigma_{U R A, i}[\mathrm{~m}]$ & $b_{\text {nom }, i}[\mathrm{~m}]$ \\
\hline 2 & $1: 00-1: 30,5: 00-5: 30$ & $1: 15-1: 30,5: 15-5: 30$ & 2.5 & $0.5,0.75,1,1.5$ \\
3 & $1: 00-1: 30,5: 00-5: 30$ & $1: 15-1: 30,5: 15-5: 30$ & $1.5,2,2.5,3$ & 0.75 \\
4 & $1: 00-4: 00$ & $1: 15-4: 00$ & 1 & 0.5 \\
\hline
\end{tabular}

Figures 2 and 3 show the HPLs computed for the DFMC SBAS and ARAIM. It can be observed that the HPLs for DFMC SBAS are generally around $5 \mathrm{~m}$ for the test periods. When the SBAS has an assumed outage, with an onset shown in the figures as black dotted line, the HPLs based on the ARAIM are used. A jump in the HPL values using ARAIM can be seen (shown in different colors for different $b_{n o m, i}$ values) compared with the HPL if SBAS continues without a break (the blue dots). It is also shown that the HPLs computed using ARAIM are strongly dependent on the parameters $\sigma_{U R A, i}$ and $b_{n o m, i}$, especially the $\sigma_{U R A, i}$, i.e. the standard deviation of the satellite clocks and orbital errors for integrity purpose. Having a $b_{\text {nom }}$ of $0.75 \mathrm{~m}$ as an example, using a $\sigma_{U R A, i}$ of $2.5 \mathrm{~m}$ as defined for horizontal ARAIM in [9] generally leads to HPLs between $10 \mathrm{~m}$ and $15 \mathrm{~m}$ for the test periods (the green dashed lines in Figure 2). When reducing $\sigma_{U R A, i}$ to $1.5 \mathrm{~m}$, as illustrated in the red lines of Figure 3, the HPLs are reduced to $5 \mathrm{~m}-10 \mathrm{~m}$.

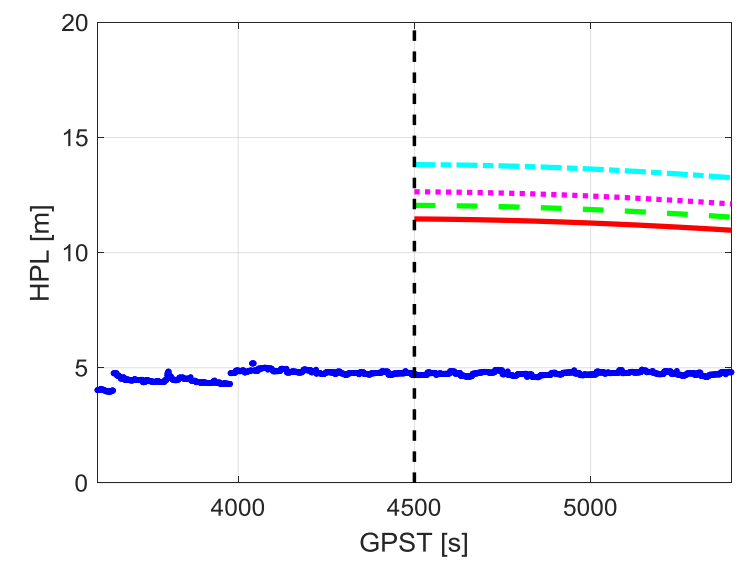

(a)

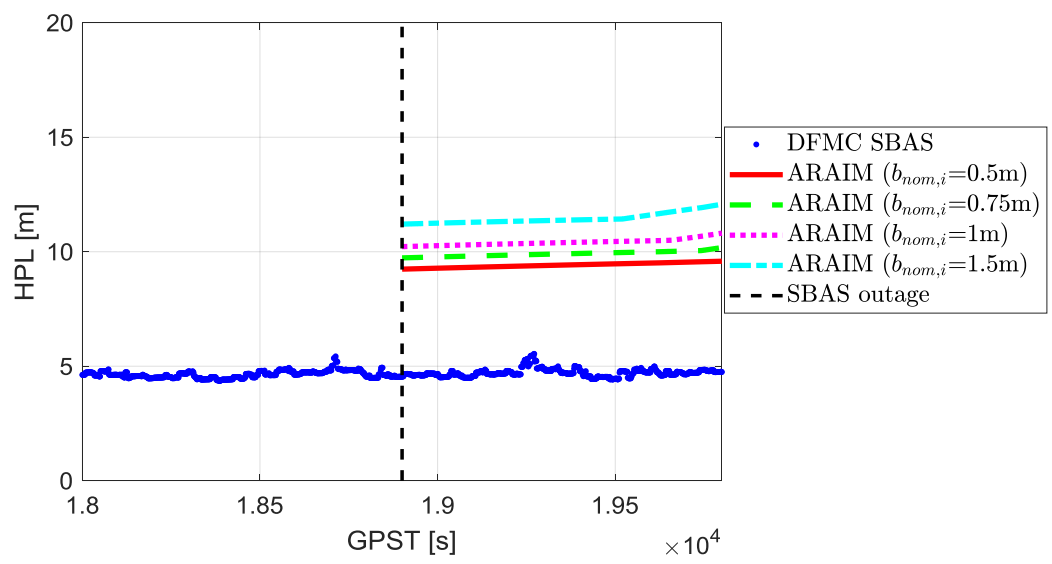

(b)

Fig 2. The HPLs computed based on the DFMC SBAS messages (blue) and the ARAIM algorithm from (a) 1:00 to 1:30 and (b) 5:00 to 5:30 in GPST on September 14, 2018. The $\sigma_{U R A, i}$ was set to $2.5 \mathrm{~m}$. 


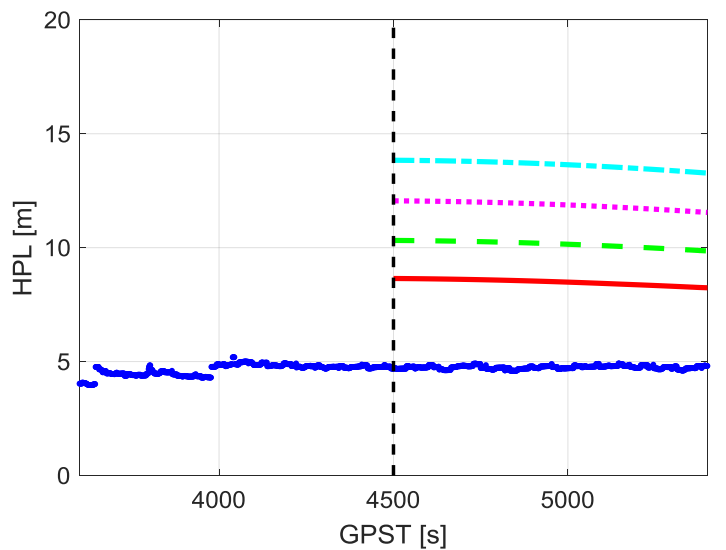

(a)

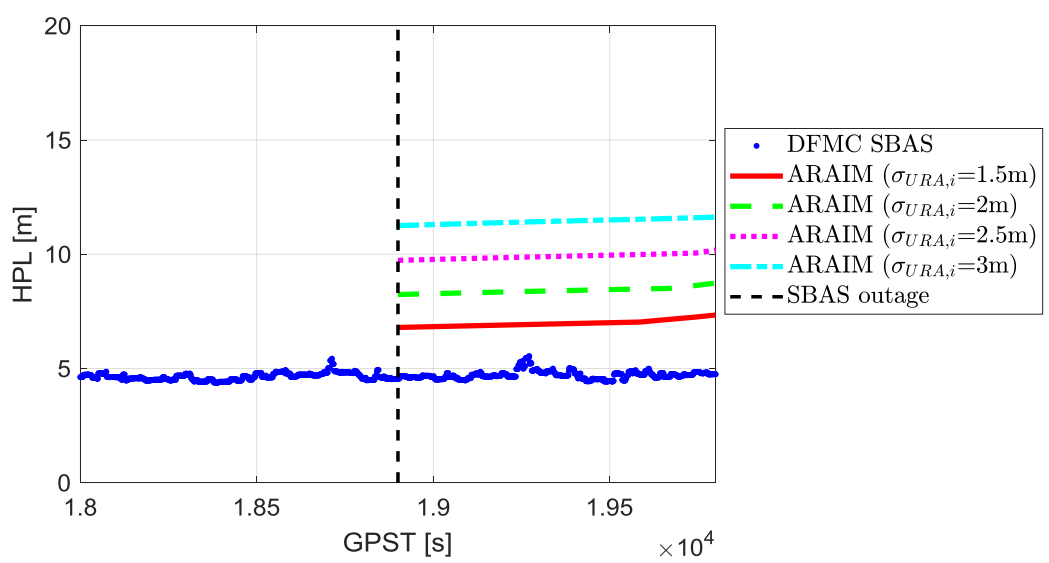

(b)

Fig 3. The HPLs computed based on the DFMC SBAS messages (blue) and the ARAIM algorithm from (a) 1:00 to 1:30 and (b) 5:00 to 5:30 in GPST on September 14, 2018. The $b_{n o m, i}$ was set to $0.75 \mathrm{~m}$.

As discussed in [10], compared with the old generation of GPS satellites, i.e., the GPS block IIA and IIR satellites, the standard deviations of the Signal-in-Space Range Error (SISRE) in the newer generation of GPS IIF satellites are decreased. This is mainly because of the improved satellite clock performance. In [17], as an example, the third constellation of GPS was assumed to have a $\sigma_{U R A, i}$ of $0.75 \mathrm{~m}$. In December 2018 and August 2019, two GPS IIIA satellites were launched and become operational in 2020. With the expectation that better accuracy can be provided in the future for the broadcasted orbits and clocks of the new GPS and Galileo satellites, an average value of $1 \mathrm{~m}$ is assumed for $\sigma_{U R A, i}$ of all satellites in the simulation to illustrate the possible reduction of the HPLs. In Figure 4, the HPLs for the ARAIM (red dots) are simulated with $\sigma_{U R A, i}$ of $1 \mathrm{~m}$ and $b_{n o m, i}$ of $0.5 \mathrm{~m}$ for the known CORS CUT0 from 1:00 to 4:00 in GPST. It can be observed that the HPLs for ARAIM (red dots) are generally at a close level to those for the DFMC SBAS (blue dots). Thus, with improved accuracy of the broadcast satellite clocks and orbits in the future, ARAIM is expected to be a useful and alternative IM approach during the SBAS outages. Yet, it should be noted that the similar HPLs of the ARAIM and DFMC SBAS are achieved under optimistic assumptions of the $\sigma_{U R A, i}$ and $b_{n o m, i}$, and about $89 \%$ of the ARAIM HPLs are still above those of the DFMC SBAS. This shows that the DFMC SBAS is still advantageous in achieving lower HPLs, and thus more availability of horizontal positioning results.

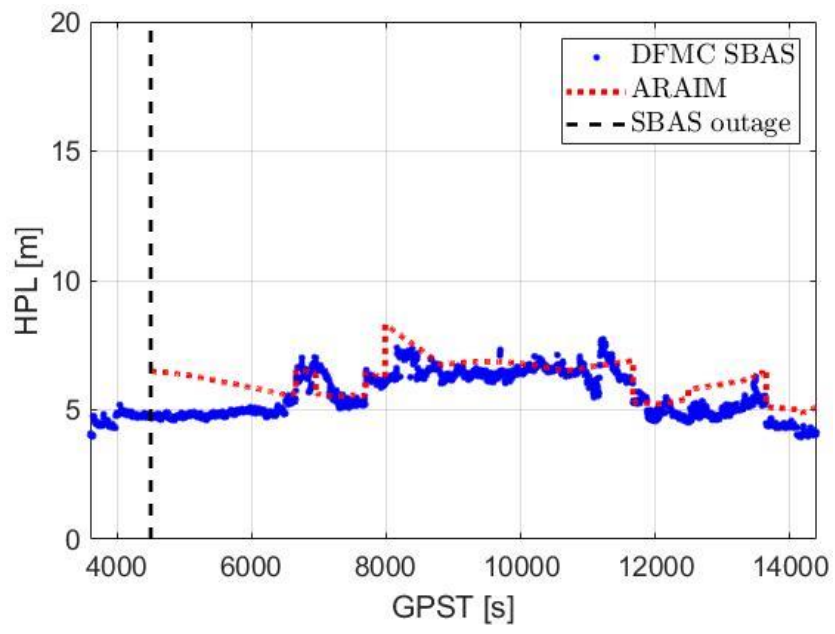

Fig 4. The HPLs computed based on the DFMC SBAS messages (blue) and the ARAIM algorithm (red) from 1:00 to 4:00 in GPST on September 14, 2018. The $\sigma_{U R A, i}$ and $b_{n o m, i}$ were set to $1 \mathrm{~m}$ and $0.5 \mathrm{~m}$, respectively.

For the road transport, different horizontal alert limits (HALs) could be implemented for different applications. With a HAL of, e.g., $5 \mathrm{~m}$, the availability of the DFMC SBAS position is about $41 \%$ for the test period in Figure 4 (see blue dots), while the ARAIM 
HPLs (see the red dashed line) are still mostly above the HAL even with the $\sigma_{U R A, i}$ and $b_{\text {nom,i }}$ set to $1 \mathrm{~m}$ and $0.5 \mathrm{~m}$, respectively. The margin of the ARAIM HPLs to the HAL of $5 \mathrm{~m}$ is, however, in such case generally within $3 \mathrm{~m}$.

\section{CONCLUSION}

The new generation of SBAS is expected to become operational soon and benefit road transport users within a few years. In addition to the traditional L1 service, the new SBAS supports the DFMC users through broadcasting precise satellite clocks, orbits, and integrity information of the GPS and Galileo satellites broadcast through the SBAS-GEO satellite via L5. Precise single-receiver positioning service can thus be realized in real time without an internet link.

In road transport, the signals from the SBAS-GEO satellite could be blocked by obstacles in the measurement environment, and possible service gap could also cause an outage of the SBAS messages. As shown in a previous study [5], the SBAS message needs to be updated regularly to avoid significant degradations in the computed protection levels. Besides, the time-out windows of diverse parameters also limit the usage of the non-updated corrections and integrity information after very short time intervals.

For bridging IM during SBAS outages, this paper utilizes a modified ARAIM algorithm to compute the HPLs, facilitating IM during this gap. The computed HPLs were compared with those based on the DFMC SBAS assuming no signal outage. The ARAIM is modelled to be consistent with SBAS methodology. Using a station located in Perth, Australia, it was shown that the ARAIM HPLs are strongly dependent on the integrity parameters $\sigma_{U R A, i}$ and $b_{n o m, i}$. With the expectation that small values of $\sigma_{U R A, i}$ and $b_{n o m, i}$ would be available in the future, having operational GPS IIIA and new Galileo satellites, the ARAIM HPLs could then reach a close level to those for the DFMC SBAS, and could thus be a useful alternative approach for the positioning integrity monitoring during SBAS outages.

\section{ACKNOWLEDGEMENTS}

The authors would like to thank GMV, Spain, for its support in providing the SBAS messages on the test day. This research is funded by the Australian Research Council (ARC) Discovery Project: Trustworthy Positioning for Next Generation Intelligent Transport Systems, Project ID: DP170103341.

\section{REFERENCES}

1. Banville, S., Collins, P., Zhang, W., Langley, R. B., "Global and Regional Ionospheric Corrections for Faster PPP Convergence", Navigation: Journal of The Institute of Navigation, Vol. 61, No. 2, 2014, pp. 115-124.

2. Leandro, R., Landau, H., Nitschke, M., Glocker, M., Seeger, S., Chen, X., Deking, A., BenTahar, M., Zhang, F., Ferguson, K., Stolz, R., Talbot, N., Lu, G., Allison, T., Brandl, M., Gomez, V., Cao, W., Kipka, A., "RTX Positioning: The Next Generation of cm-accurate Real-time GNSS Positioning", In Proc. of the ION GNSS 2011, Portland, OR, September 20-23, 2011, pp. 14601475.

3. Yu, X., Gao, J., "Kinematic Precise Point Positioning Using Multi-Constellation Global Navigation Satellite System (GNSS) Observations". ISPRS Int. J. Geo-Inf., Vol. 6, No. 1, 2017, 6.

4. Sobreira, H., Bougard, B., Barrios, J., Calle, J.D., "SBAS Australian-NZ Test Bed: Exploring New Services", Multi GNSS Asia Conference. Melbourne, Australia. 23-25 October 2018.

5. El-Mowafy, A., Wang, K., "Second Generation SBAS - Performance Analysis and Bridging Positioning and Integrity Monitoring during SBAS Outages in the Urban Environment", In Proc, of the ION GNSS+ 2019, Miami, Florida, September 16-20, 2019, pp. 2842-2854.

6. Barrios, J., Caro, J., Calle, J.D., Carbonell, E., Pericacho, J.G., Fernández, G., Esteban, V.M., Fernández, M.A., Bravo, F., Torres, B., Calabrese, A., Diaz, A., Rodríguez, I., Laínez, M.D., Romay, M.M., Jackson, R., Reddan, P.E., Bunce, D., Soddu, C., "Update on Australia and New Zealand DFMC SBAS and PPP System Results", In Proc. of the ION GNSS+ 2018, Miami, Florida, September 24-28, 2018, pp. 1038-1067.

7. EUROCAE. "Minimum operational performance standard for Galileo / Global Positioning System / Satellite-based augmentation system airborne equipment". The European organisation for civil aviation equipment, ED-259, February 2019. 
8. Blanch, J., Walter, T., Enge, P., Lee, Y., Pervan, B., Rippl, M., Spletter, A., "Advanced RAIM user algorithm description: integrity support message processing, fault detection, exclusion, and protection level calculation". In Proc. of the ION GNSS 2012, Nashville, TN, September 17-21, 2012, pp. 2828-2849.

9. ARAIM Technical Subgroup. "EU-U.S. cooperation on satellite navigation. Working group C - ARAIM technical subgroup, Milestone 3 report", Final version, February 25th, 2016.

10. Perea, S., Meurer, M., Rippl, M., Belabbas, B., Jöerger, M., "URA/SISA analysis for GPS and Galileo to support ARAIM", Navigation, Journal of the Institute of Navigation, Vol. 64, No. 2, 2017, pp. 237-254.

11. GPS SPS Performance Standard, 5th Edition (April 2020). https://www.navcen.uscg.gov/?pageName=gpsTechnicalReferences, accessed 27 April 2020.

12. El-Mowafy, A., "On detection of observation faults in the observation and position domains for positioning of intelligent transport systems", Journal of Geodesy, Vol. 93, No. 10, 2019, pp.2109-2122.

13. El-Mowafy A., Imparato, D., Rizos C., Wang J., Wang, K. "On Hypothesis Testing in RAIM Algorithms: Generalized Likelihood Ratio Test, Solution Separation Test and a Possible Alternative". Measurement Science and Technology, Vol. 30, No. 7, 075001, doi.org/10.1088/1361-6501/ab1836.

14. El-Mowafy, A., Yang, C., "Limited sensitivity analysis of ARAIM availability for LPV-200 over Australia using real data", Advances in Space Research, Vol. 57, No. 2, 2016, pp. 659-670.

15. Blanch, J., Walter, T., Enge, P., "Optimal positioning for advanced RAIM”. Navigation, Journal of the Institute of Navigation, Vol. 60, No. 4, 2013, pp. 279-289.

16. El-Mowafy, A., "ARAIM using GPS, GLONASS and Beidou”. In Proc. of the European Navigation Conference ENC-GNSS 2014, Rotterdam, Netherlands, 15-17 April, 2014.

17. Blanch, J., Walter, T., Enge, P., Wallner, S., Fernandez, F.A., Dellago, R., Ioannides, R., Hernandez, I.F., Belabbas, B., Spletter, A., Rippl, M., "Critical elements for a multi-constellation advanced RAIM". Navigation, Journal of the Institute of Navigation, Vol. 60, No. 1, 2013, pp. 53-69. 